\title{
Sensitivity Analysis to Determine the Prospect of Tollroad Investment: Case Study Krian-Legundi-Bunder-Manyar Tollroad
}

\author{
Yusuf Hartoyo and Christiono Utomo \\ Department of Business Management Technology, Institut Teknologi Sepuluh Nopember, Surabaya \\ e-mail: yusuf.hartoyo@gmail.com
}

\begin{abstract}
PT Waskita Bumi Wira as the Toll Road Business Entity (BUJT) is investing in the Krian-Legundi-Bunder-Manyar Toll road with a viability gap fund (VGF) scheme. In the 2016 Toll Road Concession Agreement (PPJT) between BPJT-BUJT-PII, several acceptable investment variables have been agreed, namely: total investment value $=\mathbf{R p} 12,224,389,000,000$, average daily traffic volume $(\mathrm{LHR})=21,874$ vehicle $/$ day, loan interest $=12.5 \%$, toll tariff for group $1=\mathbf{R p} 1,250 / \mathrm{km}$, internal rate of return on equity (IRRonEquity) $=17.64 \%$, payback period of 14 years and net present value $(\mathrm{NPV})=$ Rp. 3,731,695,000,000. During the construction, there was a design change and a delay in land acquisition, this causes cost overrun. With a concession period of 45 years, inaccurate risk factors in the prediction of traffic volume growth rates, specific and non-specific risk factors and other uncertainties greatly affect the investment prospects. Therefore investment needs to be reviewed before this toll road is divested in 2025 by sharpening the minimum attractive rate of return (MARR) and LHR variables, then comparing them with the initial business plan. The estimated selling price of the divestment is also examined in this study. The investment valuation criteria that will be used in the sensitivity analysis are NPV and IRRonEquity. The approach in determining MARR is the use of MARRpremium = $15.81 \%$. Sensitivity analysis with a deterministic approach is carried out to obtain limits on changes in investment costs, land acquisition costs, LHR traffic, loans (syndicated loans) to NPV in several tariff scenarios. This research shows that the NPV value is very sensitive to changes in investment costs. It is also known that in the toll tariff scenario according to PPJT compensation can be given in one of the conditions: investment costs increase by more than $7 \%$, LHR traffic is less than $75 \%$, or an increase in loans (syndicated loans) of more than $9 \%$. The analysis of the stochastic approach shows that the probability of not exceeding the investment budget revenue limits due to changes of $37.29 \%$ to $46.56 \%$.
\end{abstract}

Keywords-Sensitivity, IRR onEquity, MARR $_{\text {premium, tol KLBM, }}$ Stochastic.

\section{INTRODUCTION}

I N 2016 Waskita Bumi Wira company (WBW) grabbed a toll 1 road investment opportunity through initiative scheme (unsolicited) with a concession period of 45 years. This Supported Built-Operate-Transfer (BOT Supported) Project was carried out by viability gap fund (VGF) financing scheme. Based on Toll Road Concession Agreement (PPJT), WBW has a concession on 38.399 kilometers of Krian-Legundi-BunderManyar (KLBM) toll road in East Java and it obliged to construct 25 kilometers Terbanggi-Lampung with total investment was Rp 12.224.389.000.000 [1].

In terms of design, from basic design into detailed engineering design (DED) and from DED into Final Technical Plan (RTA) there are many changes in design due to the lack of detailed soil investigation data at the beginning of planning period. In terms of land acquisition, until the second year construction period (November, 2018), land acquisition progress section 1 to section 3 was $65 \%$. Meanwhile for section 4 is not acquisited yet, it affected the original construction completion in November 2018, for section 4 retreat to November 2021, while the section 1 to section 3 will be completed in May 2020. The delay in construction completion due to design and land became the affect of construction costs and investment costs increasing (cost overrun). Reduced operational period with a fixed concession period will have an effect on the KLBM toll road revenue reducing [2], [3], [4], [5], [6], [7], [8], [9], [10], [11], [12], [13], [14], [15], [16]. Therefore, the accepted limits (sensitivity) of cost variable, revenues variables and capital variables against the net present value should be re-examined before it was divested in 2025 .

\section{METHOD}

This research using minimum attractive rate of return MARR $_{\text {premium }}=15.81 \%$ and corrected rump-up period traffic.

A. Data

1. Investment cost according to PPJT and the changes in March, 2019

2. Traffic section 1 - section 4 [17]

3. Interest of debt

4. Tollrate

5. Land acquisition cost

6. Jakarta interbank offered rate (JIBOR)

*Investment cost exclude land acquisition

\section{B. Investment Criteria}

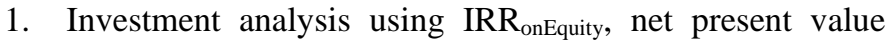
(NPV), payback period, benefit cost ratio (BCR) and profitability index (PI) 
IPTEK Proceedings Series No. (1) (2020), ISSN (2354-6026)

International Conference on Business and Engineering Management 2020 (IConBEM 2020)

February $1^{\text {st }}$ 2020, Institut Teknologi Sepuluh Nopember, Surabaya, Indonesia

Table 1.

Financial Aspects Of PPJT

\begin{tabular}{|c|c|c|c|}
\hline Number & Description & & Amount \\
\hline \multirow[t]{10}{*}{1.} & Investment cost & $\mathrm{Rp}$ & 12.224.389.000.000 \\
\hline & Design & $\mathrm{Rp}$ & 126.020.000.000 \\
\hline & Construction & $\mathrm{Rp}$ & 8.401 .374 .000 .000 \\
\hline & Tollroad tools & $\mathrm{Rp}$ & 123.278.000.000 \\
\hline & Supervision & $\mathrm{Rp}$ & 168.028.000.000 \\
\hline & Escalation & $\mathrm{Rp}$ & 1.089.376.000.000 \\
\hline & Overhead & $\mathrm{Rp}$ & 84.014 .000 .000 \\
\hline & Interest during construction (IDC) & $\mathrm{Rp}$ & 1.017.338.000.000 \\
\hline & Financial fee & $\mathrm{Rp}$ & 211.379.000.000 \\
\hline & Debt to Equity Ratio (DER) & & $70: 30$ \\
\hline 2. & Toll rate at beginning, Class I $(\mathrm{Rp} / \mathrm{km})(2018)$ & & Rp1.250 \\
\hline 3. & Tollrate escalation & & $14 \%$ per 2 years \\
\hline 4. & Traffic (vehicle/day) (2019) & & 21.874 \\
\hline 5. & Annual debt interest ( $\mathrm{i}$ loan) & & $12,50 \%$ \\
\hline 6. & Concession period (years) & & 45 \\
\hline \multirow[t]{2}{*}{7.} & $\operatorname{IRR}_{\text {onEquity }}$ (IRR with DER 2 sd 2,5) & & $17,64 \%$ \\
\hline & IRR $_{\text {onProject }}$ (IRR with $100 \%$ Equity) & & $14,59 \%$ \\
\hline 8. & Payback period & & 14 years \\
\hline 9. & Net present value (NPV) & $\mathrm{Rp}$ & 3.731 .695 .000 .000 \\
\hline
\end{tabular}

2. Analysis of sensitivity using $\operatorname{IRR}_{\text {onEquity }}$ and net present value (NPV)

\section{Variables}

Analysis of sensitivity using investment cost, land acquisition cost, all section traffic and debt (syndication loan) as the variables [18].

\section{Sensitivity Technique}

Measurement of sensitivity by using the deterministic approach sensitivity graph technique and then proceed with a stochastic approach to gain acceptance probability limits of investment.

\section{RESULTS AND DISCUSSION}

According to PPJT, the financial aspects as the investment indicator shown in Table 1.

Based on design changes, on March 2019 additional construction cost has been approved by Tollroad Regulator (BPJT) Rp 988.780.826.047 to KLBM tollroad and Rp 332.060.333.978 to VGF Terbanggi tollroad.

Additional construction cost affects value-added tax cost (PPN) dan interest during construction (IDC) thus investment cost become Rp13.833.157.828.201. The summary as shown in Table 2 and Table 3.

Ending balance sheet investment credit of KLBM tollroad with additional cost Rp 1.608.768.828.201 shown in Figure 1.

Figure 2 seen that variables influencing the NPV starting with the most influence (indicated by the graph steepest) consecutely were: investment costs, LHR traffic section 3, debt (syndication
Table 2.

KLBM Investment Cost Changes

\begin{tabular}{|c|c|c|c|c|}
\hline \multirow{2}{*}{ Number } & \multirow{2}{*}{ Description } & \multirow{2}{*}{\multicolumn{2}{|c|}{$\begin{array}{c}\text { Before } \\
\text { Amount }\end{array}$}} & \multirow{2}{*}{$\begin{array}{c}\text { After } \\
\text { Amount }\end{array}$} \\
\hline & & & & \\
\hline 1 & Design & Rp & 92.458 .000 .000 & 92.458 .000 .000 \\
\hline 2 & Construction & $\mathrm{Rp}$ & 6.163 .886 .000 .000 & $\begin{array}{ll}\text { Rp } \quad 7.152 .666 .826 .047\end{array}$ \\
\hline 3 & Tollroad tools & $\mathrm{Rp}$ & 123.278.000.000 & 123.278.000.000 \\
\hline 4 & Supervision & $\mathrm{Rp}$ & 123.278 .000 .000 & 123.278.000.000 \\
\hline 5 & Escalation & $\mathrm{Rp}$ & 804.868 .000 .000 & 804.868 .000 .000 \\
\hline 6 & Value-added tax & $\mathrm{Rp}$ & 730.777 .000 .000 & 829.654.882.605 \\
\hline 7 & Overhead & Rp & 61.639 .000 .000 & 61.639 .000 .000 \\
\hline 8 & Upfront fee & Rp & 12.774 .000 .000 & 12.774 .000 .000 \\
\hline 9 & Interest during construction & $\mathrm{Rp}$ & 748.724 .000 .000 & 865.098.278.768 \\
\hline \multirow[t]{2}{*}{10} & Financial fee & $\mathrm{Rp}$ & 155.962 .000 .000 & 155.962.000.000 \\
\hline & Investment cost & $\mathrm{Rp}$ & 9.017 .644 .000 .000 & Rp 10.221.676.987.420 \\
\hline
\end{tabular}

Table 3.

VGF Terbanggi Investment Cost Changes VGF Terbanggi Tollroad

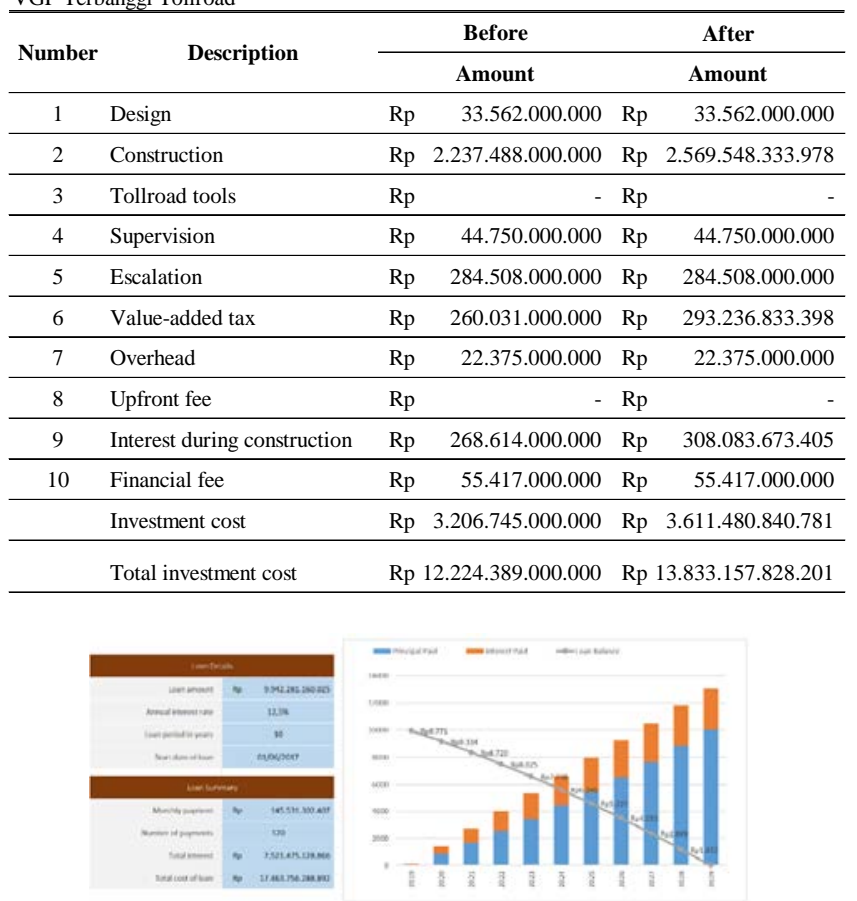

Figure 1. Ending balance sheet investment credit of KLBM tollroad with additional cost $\mathrm{Rp} 1.608 .768 .828$

loan), LHR traffic section 1, LHR traffic section 2, LHR traffic section 4 , and land acquisition. This shows that investment cost is the most sensitive to NPV. Known also that on the toll rate scenario based on PPJT the compensation fee can be given in one of the following conditions: increase of investment cost up to $7 \%$, traffic is under $75 \%$, increasing of debt (syndication loan) up to $9 \%$. These limits become the boundary whether or not the compensation from the Government to BUJT result of investment return value does not correspond to the initial agreement.

Once the limits of investment deterministically acceptance of the sensitivity analysis is obtained, further measurements of probabilities. Assuming the type of distribution can be determined based on research, interviews with experts, a data graph of the results of sensitivity analysis, assuming 
Table 4.

toll rate scenario

\begin{tabular}{|c|c|c|c|c|c|}
\hline & \multicolumn{5}{|c|}{ Toll Rate } \\
\hline & Class I & Class II & Class III & Class IV & Class V \\
\hline Toll rate $\alpha$ & Rp 1.250 & Rp 1.875 & Rp 2.500 & Rp 3.125 & Rp 3.750 \\
\hline Toll rate $\beta$ & Rp 1.250 & Rp 1.500 & Rp 2.084 & Rp 2.709 & Rp 3.104 \\
\hline Toll rate $\varphi$ & Rp 1.000 & Rp 1.500 & Rp 2.000 & Rp 2.500 & Rp 3.000 \\
\hline
\end{tabular}

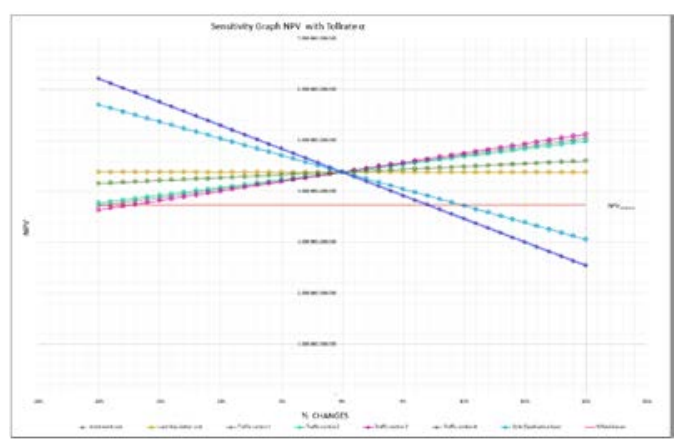

Figure 2. Sensitivity graph NPV with toll rate $\alpha$

Table 5.

Assumption of Probability Distribution Function

\begin{tabular}{|c|c|c|c|}
\hline Uncertainty variable & $\begin{array}{c}\text { Probability } \\
\text { distribution } \\
\text { funcion }\end{array}$ & Parameter & Note \\
\hline Investment cost & Normal & $\begin{array}{c}\text { Mean } \mu \\
\text { Standar deviasi } \sigma \\
\text { Static value }\end{array}$ & $\begin{array}{l}\mu=\operatorname{Rp} 13.833 .157 .828 .201 \\
107 \%=\operatorname{Rp} 14.801 .476 .876 .175 \\
\sigma=\operatorname{Rp} 968.319 .047 .974 \\
\text { static value }=\mu\end{array}$ \\
\hline $\begin{array}{l}\text { Land acquisition } \\
\text { cost }\end{array}$ & Normal & $\begin{array}{c}\text { Mean } \mu \\
\text { Standar deviasi } \sigma \\
\text { Static value }\end{array}$ & $\begin{array}{l}\mu=\operatorname{Rp} 1.371 .355 .604 .273 \\
+120 \%=\operatorname{Rp} 1.645 .626 .725 .127 \\
\sigma=\operatorname{Rp} 274.271 .120 .854 \\
\text { static value }=\mu\end{array}$ \\
\hline Traffic section 1 & Normal & $\begin{array}{c}\text { Mean } \mu \\
\text { Standar deviasi } \sigma \\
\text { Static value }\end{array}$ & $\begin{array}{l}\mu=1.368 .140 .931 \text { vehicle } \\
-81 \%=1.108 .194 .154 \text { vehicle } \\
\sigma=259.946 .777 \text { vehicle } \\
\text { static value }=\mu\end{array}$ \\
\hline Traffic section 2 & Normal & $\begin{array}{c}\text { Mean } \mu \\
\text { Standar deviasi } \sigma \\
\text { Static value }\end{array}$ & $\begin{array}{l}\mu=1.358 .602 .150 \text { vehicle } \\
-79 \%=1.073 .295 .698 \text { vehicle } \\
\sigma=285.406 .452 \text { vehicle } \\
\text { static value }=\mu\end{array}$ \\
\hline Traffic section 3 & Normal & $\begin{array}{c}\text { Mean } \mu \\
\text { Standar deviasi } \sigma \\
\text { Static value }\end{array}$ & $\begin{array}{l}\mu=1.373 .634 .470 \text { vehicle } \\
-83 \%=1.140 .116 .610 \text { vehicle } \\
\sigma=233.517 .860 \text { vehicle } \\
\text { static value }=\mu\end{array}$ \\
\hline Traffic section 4 & Normal & $\begin{array}{c}\text { Mean } \mu \\
\text { Standar deviasi } \sigma \\
\text { Static value }\end{array}$ & $\begin{array}{l}\mu=637.280 .204 \text { vehicle } \\
-42 \%=267.657 .686 \text { vehicle } \\
\sigma=369.622 .518 \text { vehicle } \\
\text { static value }=\mu\end{array}$ \\
\hline Syndication loan & Normal & $\begin{array}{c}\text { Mean } \mu \\
\text { Standar deviasi } \sigma \\
\text { Static value }\end{array}$ & 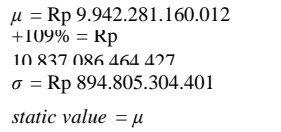 \\
\hline
\end{tabular}

subjectively based on the preferences of researchers, and others. Based on the sensitivity analysis of data in Figure 2 Sensitivity graph NPV with toll rate $\alpha$ curve is a straight line formed then subjectively all the variables under review are assumed to be normally distributed as shown in Table 5 below :

From the simulation with a confidence level of $95 \%(\alpha=.05)$ and with 100,000 iterations obtained probability investment

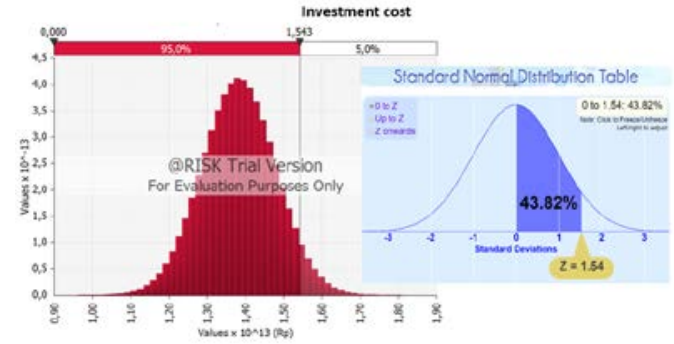

Figure 3. Probability density function of investment cost

costs increased to a maximum of $107 \%$ to $\mathrm{Rp}$ 14.801.476.876.175 is $43.82 \%$ as shown in Figure 3 below:

From analysis through stochastic approach provides information that probability sensitivity investment cost = $43.82 \%$, probability sensitivity acquisition costs $=46.56 \%$, probability sensitivity traffic section $1=46.33 \%$, section $2=$ $46.56 \%$, section $3=45.99 \%$, section $4=39.25 \%$ and probability sensitivity syndication loan $=37.29 \%$.

\section{REFERENCES}

[1] Notaris Pejabat Pembuat Akta Tanah (PPAT), Salinan Akta Perjanjian Pengusahaan Jalan Tol Krian-Legundi-Bunder-Manyar. Jakarta, 2016.

[2] Y. L. D. Denny Abdurachman and A. Wibowo, "STRUKTUR MODAL OPTIMAL DALAM ANALISIS KELAYAKAN FINANSIAL PROYEK JALAN TOL.”

[3] Y. Agustina, "Analisa Studi Kelayakan Jalan Tol Krian-Legundi-BunderManyar Seksi I \& II,” Institut Teknologi Sepuluh Nopember, 2017.

[4] D. Emeralda, Y. Rivina, and A. Dhiandini, "Evaluasi Kelayakan Pembiayaan Pembangunan Jalan Tol Palembang - Indralaya.” 2014.

[5] H. Fitriani, P. Farida, and A. Wibowo, "Kajian penerapan model NPV-atrisk sebagai alat untuk melakukan evaluasi investasi pada proyek infrastruktur jalan tol,” J. Infrastruktur dan Lingkung. Binaan, vol. 2, no. 1, pp. 1-12, 2006.

[6] L. S. Hidayat, "Studi Kelayakan Pembangunan Jalan Tol Krian-LegundiBunder-Manyar Seksi III dan IV,” Institut Teknologi Sepuluh Nopember, 2017.

[7] S. C. Kusuma, "Analisis Dampak Keterlambatan Pengadaan Tanah Dan Pelaksanaan Konstruksi Terhadap Masa Konsesi Pada Pembangunan Jalan Tol Kertosono-Mojokerto,” Institut Teknologi Sepuluh Nopember, 2017.

[8] D. Listyaningsih and C. Utomo, "Pengaruh Keterlambatan Pembebasan Lahan Terhadap Keputusan Investasi Proyek Jalan Tol SurabayaMojokerto,” J. Tek. ITS, vol. 3, no. 2, pp. E44--E46, 2014.

[9] I. Mahani, R. Z. Tamin, K. S. Pribadi, and A. Wibowo, "Evaluation of implementation viability gap funding (VGF) policy on toll road investment in Indonesia," in AIP Conference Proceedings, 2017, vol. 1903, no. 1, p. 70013.

[10] A. P. JF, "ANALISIS RISIKO FINANSIAL INVESTASI JALAN TOL AKIBAT PERUBAHAN INFLASI,” J. Momentum, vol. 18, no. 2, 2016.

[11] K. Rahardjo, "STUDI KELAYAKAN INVESTASI JALAN TOL SEGMEN LAWANG-PURWOSARI,” J. Spectra, vol. 5, no. 09, pp. 1624, 2017.

[12] A. Rahmalia, F. A. Hasani, D. Purwanto, and W. Kushardjoko, "Analisa Kelayakan Pembangunan Jalan Tol Pemalang Batang,” J. Karya Tek. Sipil, vol. 5, no. 1, pp. 11-24, 2016.

[13] D. San Santoso, T. B. Joewono, A. Wibowo, H. P. A. Sinaga, and W. Santosa, Public-private partnerships for tollway construction and operation: Risk assessment and allocation from the perspective of investors. 2012.

[14] I. Sopiana, "Analisis investasi pembangunan jalan Tol Waru-Bandara Juanda (studi kasus pada PT XYZ),” Widya Mandala Catholic University Surabaya, 2008.

[15] D. Susilo and C. Utomo, "ANALISIS KELAYAKAN FINANSIAL PEMBANGUNAN JALAN TOL GEMPOL-PANDAAN."

[16] A. Wibowo, "Inflasi dalam Analisis Finansial Investasi Jalan Tol: 
International Conference on Business and Engineering Management 2020 (IConBEM 2020)

February $1^{\text {st }}$ 2020, Institut Teknologi Sepuluh Nopember, Surabaya, Indonesia Perlakuan dan Pengaruhnya bagi Badan Usaha dan Pemerintah,” J. Tek. Sipil, vol. 19, no. 1, pp. 15-24, 2012.

[17] W. Putro, Analisa Mengenai Dampak Lingkungan Jalan Tol KrianLegundi-Bunder-Manyar. PT Metro Karya Inditama. Surabaya, 2016.

[18] A. Alfian, “Analisa Sensitivitas Pertumbuhan Lalu Lintas Dan Probabilitas Risiko Pada Pembangunan Jalan Tol Kategori Priority Project,” J. Tek. Sipil, vol. 12, no. 3, 2016. 